\title{
选择性外延生长大尺寸二硫化铇/二硫化锄横向和 垂直异质结
}

孙研 ${ }^{1}$, 王琳 ${ }^{2,3^{*}}$, 黄维 $1,2,3^{*}$

1. 西北工业大学柔性电子前沿科学中心, 西安 710072 ;

2. 南京邮电大学有机电子与信息显示国家重点实验室, 信息材料与纳米技术研究院, 南京 210023 ;

3. 南京工业大学柔性电子国家重点实验室培育建设点, 先进材料研究院, 南京 211816

* 联系人, E-mail: iamlwang@njtech.edu.cn; iamwhuang@nwpu.edu.cn

\section{Selective epitaxial growth of large $\mathrm{WS}_{2} / \mathrm{MoS}_{2}$ lateral and vertical heterostructures}

\author{
Yan Sun ${ }^{1}$, Lin Wang ${ }^{2,3^{*}}$ \& Wei Huang ${ }^{1,2,3^{*}}$ \\ ${ }^{1}$ Frontiers Science Center for Flexible Electronics, Northwestern Polytechnical University, Xi'an 710072, China; \\ ${ }^{2}$ State Key Laboratory of Organic Electronics and Information Displays \& Institute of Advanced Materials, Nanjing University of Posts \& Telecommu- \\ nications, Nanjing 210023, China; \\ ${ }^{3}$ Key Laboratory of Flexible Electronics \& Institute of Advanced Materials, Nanjing Tech University, Nanjing 211816, China \\ * Corresponding authors, E-mail: iamlwang@njtech.edu.cn; iamwhuang@nwpu.edu.cn \\ doi: 10.1360/TB-2020-1442
}

过渡金属硫化物因其优异的电学、光学性能和良好的 机械柔韧性引起了人们的广泛关注 ${ }^{[1]}$. 但是, 单一的材料 由于能带和导电性等物理性质的局限性, 不能满足更多的 器件需求 ${ }^{[2,3]}$. 因此, 二维过渡金属硫化物异质结的制备显 得尤为重要. 目前制备二维异质结主要有两种方法, 一种 是机械转移法, 直接将两种材料通过机械剥离和转移的方 式堆叠在一起形成垂直异质结 ${ }^{[4]}$; 另一种方法是化学气相 沉积法, 将不同的金属源气化沉积在基底表面反应生长横 向或者垂直异质结 ${ }^{[5]}$. 由于成核位点的不可控, 很难实现 横向和垂直异质结的选择性生长 ${ }^{[6]}$. 另外, 由于合金具有 更高的配置熵，在异质结界面处很容易出现金属掺杂而产 生合金, 影响异质结的质量 ${ }^{[7]}$. 因此, 高质量 $\mathrm{WS}_{2} / \mathrm{MoS}_{2}$ 横 向和垂直异质结的选择性生长一直是研究的难点.

针对以上的难点, 苏州大学邹贵付课题组 ${ }^{[8]}$ 在Journal of the American Chemical Society上发表了题为“Onepot selective epitaxial growth of large $\mathrm{WS}_{2} / \mathrm{MoS}_{2}$ lateral and vertical heterostructures”的文章, 利用氢氧辅助法在不同退 火温度下实现了高质量 $\mathrm{WS}_{2} / \mathrm{MoS}_{2}$ 横向和垂直异质结的选 择性生长, 其中, 横向异质结尺寸可达 $1 \mathrm{~mm}$, 是目前报道 的最大横向异质结尺寸. 通过高角环形暗场扫描透射电子 显微镜(HAADF-STEM) 证明了生长得到的异质结具有清
晰平整的异质结界面，没有合金的产生. 另外，利用飞行 时间二次离子质谱仪(ToF-SIMS)和理论计算对异质结选 择性生长机理进行了系统性的研究.

他们将金属Mo和W源溶解在含有大量氢氧根离子的 水中, 然后旋涂在蓝宝石基底表面. 在高温退火过程中, 由于氢氧根离子吸附在单层 $\mathrm{MoS}_{2}$ 表面, 能够有效地阻止 金属 $\mathrm{W}$ 在 $\mathrm{MoS}_{2}$ 表面成核生长, 促进 $\mathrm{WS}_{2}$ 在 $\mathrm{MoS}_{2}$ 的边缘外 延生长并形成 $\mathrm{WS}_{2} / \mathrm{MoS}_{2}$ 横向异质结. 退火温度达到 $850^{\circ} \mathrm{C}$ 后, $\mathrm{MoS}_{2}$ 表面的氢氧根离子在高温下被破坏, $\mathrm{WS}_{2}$ 由于更 高的扩散势垒在 $\mathrm{MoS}_{2}$ 表面成核, 形成 $\mathrm{WS}_{2} / \mathrm{MoS}_{2}$ 垂直异质 结. HAADF-STEM观察到 $\mathrm{WS}_{2} / \mathrm{MoS}_{2}$ 横向和垂直异质结中 原子级平整的异质界面，没有出现金属 Mo和W的相互取 代, 证明异质结具有很高的质量. 同时, 选区电子衍射图 证明了两种异质结材料的外延生长. 另外, 他们通过 ToF-SIMS直接观察到 $\mathrm{WS}_{2} / \mathrm{MoS}_{2}$ 水平异质结中的 $\mathrm{MoS}_{2}$ 表面 吸附的大量氢氧根离子(图 1), 而在 $\mathrm{WS}_{2} / \mathrm{MoS}_{2}$ 垂直异质结 中的 $\mathrm{MoS}_{2}$ 表面没有观察到氢氧根离子的存在. 研究结果 证明氢氧根离子对于异质结选择性生长具有关键作用. 将 得到的单层 $\mathrm{MoS}_{2}-\mathrm{OH}$ 和两种结构的异质结制备了半导体 场效应管, 其中 $\mathrm{WS}_{2} / \mathrm{MoS}_{2}$ 水平异质结具有最高的载流子 迁移率 $\left(58 \mathrm{~cm}^{2} \mathrm{~V}^{-1} \mathrm{~s}^{-1}\right)$ 和开关比 $\left(10^{8}\right)$, 进一步证明了该方 
(a)

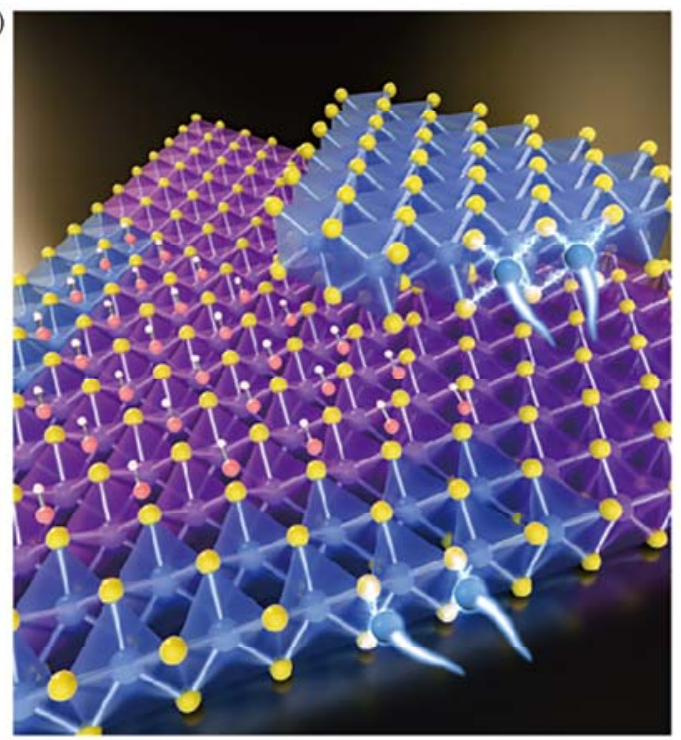

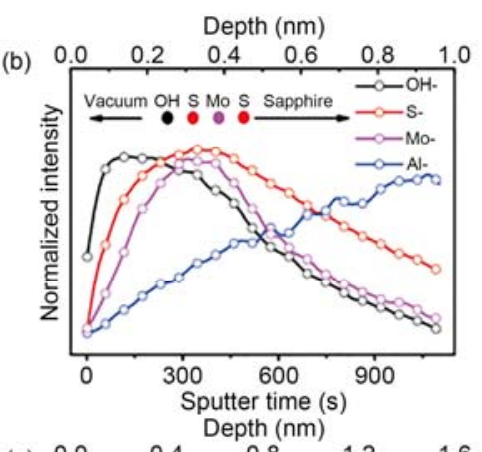

(c) 0.0

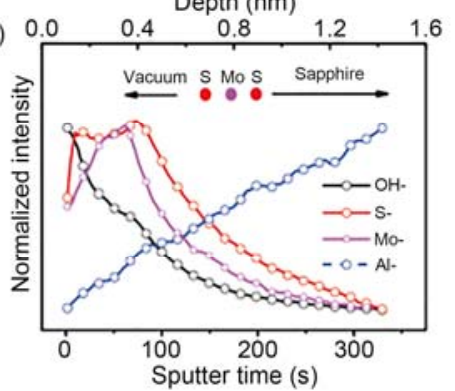

图 1 (网络版彩色) $\mathrm{WS}_{2} / \mathrm{MoS}_{2}$ 横向和垂直异质结的结构示意图(a)以及两种异质结中 $\mathrm{MoS}_{2}$ 的ToF-SIMS图(b,c)

Figure 1 (Color online) Atomic structures (a) and ToF-SIMS depth profile (b, c) of the lateral and vertical $\mathrm{WS}_{2} / \mathrm{MoS}_{2}$ heterostructures

法生长得到高质量的异质结.

邹贵付课题组通过氢氧根离子辅助法实现了大面积 高质量的 $\mathrm{WS}_{2} / \mathrm{MoS}_{2}$ 横向和垂直异质结的选择性生长, 生
长得到的异质结具有清晰平整的异质界面，具有优异的电 学性能. 这种原子级厚度的高质量二维异质结材料在微型 光电子器件领域具有巨大的应用潜力.

\section{参考文献}

1 Wang Z, Jingjing Q, Wang X, et al. Two-dimensional light-emitting materials: Preparation, properties and applications. Chem Soc Rev, 2018, 47: 6128-6174

2 Zhang Z, Chen P, Duan X, et al. Robust epitaxial growth of two-dimensional heterostructures, multiheterostructures, and superlattices. Science, 2017, 357: 788-792

3 Sun Y, Zhou Z, Huang Z, et al. Band structure engineering of interfacial semiconductors based on atomically thin lead iodide crystals. Adv Mater, 2019, 31: 1806562

4 Kang D H, Pae S R, Shim J, et al. An ultrahigh-performance photodetector based on a perovskite-transition-metal-dichalcogenide hybrid structure. Adv Mater, 2016, 28: 7799-7806

5 Gong Y, Lin J, Wang X, et al. Vertical and in-plane heterostructures from $\mathrm{WS}_{2} / \mathrm{MoS}_{2}$ monolayers. Nat Mater, 2014, 13: 1135-1142

6 Zhou J, Tang B, Lin J, et al. Morphology engineering in monolayer $\mathrm{MoS}_{2}-\mathrm{WS}_{2}$ lateral heterostructures. Adv Funct Mater, 2018, 28: 1801568

7 Zhang Q, Xiao X, Zhao R, et al. Two-dimensional layered heterostructures synthesized from core-shell nanowires. Angew Chem Int Ed, 2015, 127: 9085-9088

8 Zhu J, Li W, Huang R, et al. One-pot selective epitaxial growth of large $\mathrm{WS}_{2} / \mathrm{MoS}_{2}$ lateral and vertical heterostructures. J Am Chem Soc, 2020, 142: 16276-16248 